\title{
Sustentabilidad universitaria en México: avances y desafíos
}

University sustainability in Mexico: progress and challenges

Sustentabilidade universitária no México: avanços e desafios

Nancy Merary Jiménez-Martínez/ Universidad Nacional Autónoma de México, México/ nancy.merary@gmail.com

Recibido: 22/4/2021

Aceptado: 27/7/2021

Publicado: $24 / 8 / 2021$

\section{RESUMEN}

La sustentabilidad Ilama a una transformación profunda de la universidad. Este artículo de revisión tuvo como propósito examinar cómo ha sido la adopción de la sustentabilidad en las universidades mexicanas e identificar sus avances y desafíos. Se revisaron los artículos publicados en nueve revistas mexicanas cuyo contenido se refiere a la sustentabilidad, la sostenibilidad, la educación ambiental, el ambiente o la gestión ambiental en la educación superior en México. Tres fueron las dimensiones consideradas para abordar la sustentabilidad en la universidad: universidad integral, universidad integrada y universidad integradora. Los resultados obtenidos mostraron que los avances más sólidos se tuvieron en las dos primeras dimensiones; de ello da cuenta la sostenibilización curricular y el enverdecimiento del campus. Asimismo, se determinó que los principales desafíos radican en la dimensión «universidad integradora», específicamente, en la falta de construcción de una agenda política de sustentabilidad por parte del gobierno universitario.

Palabras clave: campus universitario, currículo universitario, desarrollo sustentable, manejo ambiental universitario 


\begin{abstract}
A deeper understanding of sustainability calls for a profound transformation of university program curriculums. The purpose of this review was to examine how the adoption of sustainability has been explored in Mexican universities, and to identify its progress and challenges. Articles published in nine Mexican journals whose content refers to sustainability and environmental education, the environment or environmental management within higher education in Mexico was reviewed. Three of these reviews were the dimensions considered to address sustainability in the university: comprehensive university, integrated university and inclusive university. The results obtained showed that the most solid advances were made in the first two dimensions; This is reflected in the sustainability of the curriculum and the greening of the campus. Likewise, it was determined that the main challenges lie in the "inclusive university" dimension, specifically, in the lack of construction of a political agenda for sustainability by the university authorities.
\end{abstract}

Keywords: sustainable development, university campus, university curriculum, university environmental management

\title{
RESUMO
}

A sustentabilidade requer uma profunda mudança da universidade. E este artigo de revisão, teve como objetivo buscar como tem sido a adaptação da sustentabilidade nas universidades do México, além de identificar seus avanços e desafios. Foram revisados artigos publicados em nove revistas mexicanas no qual o conteúdo cientifico se baseava em sustentabilidade, viabilidade, educação ambiental, ambiente ou gestão ambiental na educação superior do México. Diante disso, considerou-se três dimensões para abordar sobre sustentabilidade dentro da universidade, tais como universidade integral, universidade integrada e universidade integradora. Observou-se como resultado, que os avanços mais significativos estiveram nas duas primeiras dimensões, isto reflete na viabilidade curricular e na ambientalização do campus universitário. Portanto, foi também determinado que os principais desafios se encontram na dimensão "universidade integradora", especificamente, na falta de construção de políticas de sustentabilidade pelo governo para as universidades.

Palavras chave: campus universitário, currículo universitário, desenvolvimento sustentável, gestão ambiental universitária

\section{INTRODUCCIÓN}

Desde la Cumbre de la Tierra en Estocolmo, se señaló que las universidades tienen un compromiso especial en el desarrollo sustentable. El Ilamado que la sustentabilidad hace a la universidad actualiza su papel estratégico en el desarrollo de la sociedad, restablece su significado y función, y enfatiza su papel como microcosmos de referencia para la comunidad en general. Se espera que la universidad responda con productos, 
políticas y modelos que reflejen su ethos, el de una comunidad donde acuden «los sujetos que de manera voluntaria se relacionan en torno al saber..., donde se transmite, se crea y se certifica el saber..., y que extiende su labor e influencia más allá de sus fronteras»(Bravo, 2006, p. 91).

La sustentabilidad universitaria se ha difundido fuertemente desde finales de los ochenta, a partir de esfuerzos como el de la Carta de Bogotá sobre Medio Ambiente y Universidad (1985), la Declaración de Talloires (1990), la Declaración de Swansea (1993), la Declaración CRE-Copernicus (1994), la Declaración de Lüneburg (2001) y la Década de las Naciones Unidas de la Educación para el Desarrollo Sustentable (2005), entre otros. Estas iniciativas representan intentos por convertir a la sustentabilidad en una dimensión fundamental en la vida de estas instituciones, capaces de impregnar su estructura, modificar sus contenidos, enfoques y prácticas, y orientar su acción hacia la solución de los problemas medioambientales.

Cantú-Martínez (2013) puntualiza cuatro aspectos para desarrollar la sustentabilidad en la universidad. El primero es la gestión interna, que se refiere a la transformación intrínseca de la institución con miras a convertirse en una comunidad basada en la democracia, la equidad y la transparencia, e impulsora del desarrollo sustentable. El segundo es la docencia, desde la cual se promoverá el aprendizaje sustentado en proyectos sociales y aplicado a resolver pro- blemas, así como la capacitación a la planta académica. El tercero es la investigación, que fomentará la vinculación interdisciplinaria y la orientación hacia problemáticas de la colectividad social. El cuarto es la proyección social, que conjuga los elementos anteriores y los encamina hacia proyectos de desarrollo.

De lo anterior se desprende que la sustentabilidad estimula a la universidad a modificar sus principios organizacionales, sus contenidos curriculares y sus enfoques de investigación; recuperar las mejores tradiciones de su comunidad para transformar la realidad socioambiental, y autoreformarse para transitar hacia una universidad integral, que alinee sus funciones sustantivas hacia el desarrollo sustentable; integrada, que atienda las necesidades de su contexto, e integradora, que transite hacia formas más amplias de gobierno (Suasnábar et al., 2018).

Se retoman los planteamientos de Cantú-Martínez (2013) y de Suasnábar et al. (2018) quienes definen una universidad sustentable como aquella que es integral, donde las tareas sustantivas de investigación, docencia y transmisión de la cultura están orientadas al desarrollo sustentable. Es integrada, porque sus capacidades se encaminan a resolver los problemas de su entorno inmediato a través del desarrollo de proyectos, e integradora, ya que sus actividades de gestión y gobierno revelan que esta se rige por la democracia, la equidad y la transparencia. Lo anterior se presenta en la figura 1. 
Figura 1. Dimensiones de la universidad sustentable

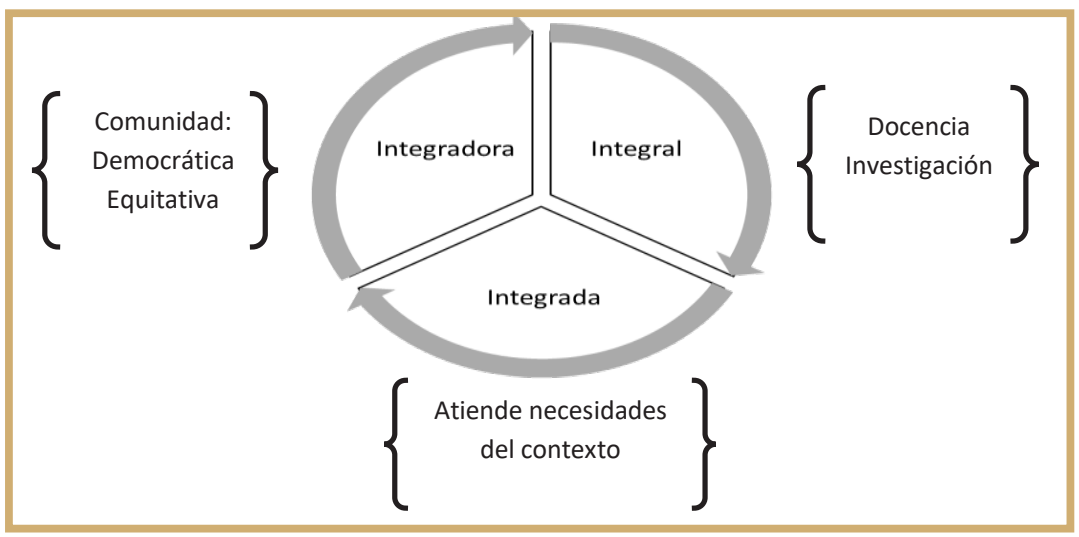

Fuente: Elaboración propia sobre la base de Cantú-Martínez (2013) y Suasnábar et al. (2018).

A partir de una revisión documental, se examinó cómo ha sido la adopción de la sustentabilidad en las universidades mexicanas y se identificó en qué dimensiones se muestran avances y en cuáles se enfrentan obstáculos. Acudiendo al Sistema de Clasificación de Revistas Mexicanas de Ciencia y Tecnología, se acotó la búsqueda a las revistas de ciencias sociales y, dentro de estas, se seleccionaron las enfocadas en las temáticas de educación, procesos de enseñanza y aprendizaje, investigación educativa para la educación superior, y estudios socioambientales ${ }^{1}$. La selección de artículos se limitó a aquellos que respondieron a las palabras sustentabilidad, sostenibilidad, educación ambiental, medioambiente y gestión ambiental en la educación superior en México. No se tuvo ninguna restricción temporal.

\section{La sustentabilidad universitaria y sus líneas de investigación}

En las ciencias sociales se identifican tres líneas de investigación que abordan la sustentabilidad universitaria. La primera se ha orientado a documentar el enverdecimiento de las operaciones del campus (Carlson, 2015), que se expresa en el desarrollo de prácticas ambientales en las tareas cotidianas, tales como la disminución del consumo energético y del agua, la separación de residuos, y la movilidad, como en los esfuerzos por institucionalizar la sustentabilidad y establecer nuevas relaciones entre la comunidad universitaria y su medioambiente. Este rubro ha avanzado con el establecimiento de Sistemas de Gestión Medioambiental (Barnes y Jerman, 2002).

${ }^{1}$ Revistas seleccionadas: Revista de la Educación Superior ANUIES; Perfiles Educativos; Revista de Investigación Educativa de la REDIECH; Revista Electrónica de Investigación Educativa; Revista Iberoamericana de Educación Superior; Revista Mexicana de Investigación Educativa; Sinéctica: Revista Electrónica de Educación; Regiones y Desarrollo Sustentable, y Sociedad y Ambiente. 
La segunda línea aborda las prácticas y las percepciones del alumnado y del profesorado hacia la sustentabilidad (Porras, 2015; Harring et al., 2017). En esta se registran debates y diferencias entre perfiles y entre campus universitarios (Barnes y Jerman, 2002; Ortiz et al., 2019), así como reflexiones en torno a la forma en que se integra la perspectiva ambiental en determinadas formaciones (Saucedo, 2015).

La tercera línea se refiere a la ambientalización del currículo universitario y estudia la incorporación de la sustentabilidad como disciplina académica o programa de estudios para formar perfiles profesionales con prácticas transformadoras basadas en una nueva ética ambiental (Ortiz, 2019; Pedraza, 2020). La también denominada "sostenibilización curricular» constituye la dimensión predominante de estudio de la sustentabilidad universitaria. En esta línea se discuten los retos que se enfrentan en la formación de profesionistas y las competencias profesionales para la sustentabilidad (Mora, 2015), así como las estrategias para la ambientalización del currículo y las políticas institucionales para llevarla a cabo (Fuentes y González, 2016; Pedraza, 2017).

Lo anterior muestra que, aunque la sustentabilidad busca transformar profundamente a las universidades para luego extender dicha transformación a la sociedad, los avances no han sido integrales.

\section{Avances de la sustentabilidad en la educa- ción superior en México}

En México, la ambientalización curricular ha sido uno de los ámbitos más desarrollados. Sáenz (2015) establece una relación de los numerosos esfuerzos que se han hecho en este campo: Estudio sobre la incorporación de la Formación Ambiental a la Educación Superior (1984); Programa Nacional de Educación Ambiental (1986), del cual derivaron las primeras investigaciones en educación ambiental; Oferta Educativa de Estudios Ambientales en Instituciones de Educación Superior en México (1993); Estado de la Investigación en Educación Ambiental en las Instituciones de Educación Superior en México (1994); Avances en el campo de la investigación en la educación ambiental en México (2000); Acciones Ambientales de las Instituciones de Educación Superior en México en la perspectiva del desarrollo sustentable: antecedentes y situación actual, que recoge iniciativas que abonan a la comprensión de aspectos académicos, institucionales y de gestión de las Instituciones de Educación Superior (IES); Educación Ambiental en México: Logros, Perspectivas y Retos de Cara al Nuevo Milenio, en el cual se señala el notable incremento de los programas académicos en la década de los noventa.

Para Sáenz y Benayas (2015), este incremento en la oferta educativa «en el área de estudios ambientales fue explosivo» ( $p$. 198), tanto por la incorporación de materias ambientales en el currículo como por la creación de nuevas profesiones y posgrados relacionados con la sustentabilidad ${ }^{2}$ (Ávila, 2014; Martínez-Fernández y González, 2015). No obstante, los avances de la «sostenibilización curricular» reportan dos limi-

${ }^{2}$ Martínez-Fernández y González (2015) señalan que se pasó de 290 programas de estudio en 1993 a 1399 en 2001. 
tantes: por una parte, aunque se reconoce que la inclusión de asignaturas ambientales en los currículos de programas académicos es un avance, este es claramente insuficiente (Fuentes y González, 2016). Asimismo, se advierte que la oferta de profesionalización de los educadores ambientales es escasa ${ }^{3}$ (Anzueto, 2015).

Por otra parte, la incorporación de «lo ambiental» manifiesta una falta de transversalidad, cuyo resultado ha sido, o bien su anexión desarticulada en la formación profesional, o bien su concentración en disciplinas «afines» (Martínez-Fernández y González, 2015), pero no una reflexión que genere aprendizajes significativos y conduzca a la comprensión en su complejidad de la crisis ecológica y socioeconómica que experimentamos (González y Meira, 2020; González y Arias, 2009; Rosas et al., 2015; Ávila, 2014). También se reporta que "no siempre las nuevas actividades de formación estuvieron acompañadas de las correspondientes acciones de investigación y extensión» (Sáenz y Benayas, 2015, p. 205), lo que sugiere que la división disciplinaria y el distanciamiento entre estas impiden la adopción transversal de la sustentabilidad (Pedraza, 2017; Ortiz, 2019).

La investigación en educación ambiental se ubica estrechamente vinculada con este campo. En México esta es un área consolidada (Calixto, 2012) desde la cual se ha evaluado la producción científica de la susten- tabilidad ambiental (Celaya et al., 2017), los temas más influyentes en la investigación sobre la sostenibilidad ambiental universitaria y la relación que existe entre ellos (Miranda et al., 2021). A esta línea de investigación se la identifica, debido a su carácter educativo, como un tipo de investigación con una identidad propia, cuya agenda se centra en identificar problemas, áreas de oportunidad y perspectivas de desarrollo (Calixto, 2012).

El análisis de las prácticas y percepciones de la sustentabilidad universitaria es un área de investigación de reciente atención. En este ámbito destacan las investigaciones sobre las representaciones sociales de la problemática ambiental entre los diferentes sectores universitarios, con un énfasis en el sector académico (Ortiz et al., 2019) y sus percepciones frente a la ambientalización curricular (Sánchez-Contreras y Murga-Menoyo, 2019). Otra línea de estudios se centra en documentar la percepción ambiental y de sostenibilidad en los estudiantes (Martínez y Juárez, 2019), así como en las representaciones sociales del cambio climático en este sector de la comunidad universitaria (Calixto, 2018), e identificar los factores que alientan o constriñen su participación en iniciativas ambientales dentro del campus universitario (Prado y Pérez, 2010).

El enverdecimiento del campus es un elemento que se ha desarrollado ampliamente y ha estado articulado con la implementación de sistemas de gestión ambientales. Las

${ }^{3}$ De acuerdo con datos de la Asociación Nacional de Instituciones de Educación Superior (2020) del ciclo escolar (2019-2020), la oferta académica de las IES para la profesionalización de los educadores ambientales es restringida, conformada por 24 licenciaturas y 19 maestrías. 
raíces de estas iniciativas se encuentran en el fomento de los planes ambientales institucionales (PAI) a finales de los noventa, luego que el Plan de acción para el desarrollo sustentable en las instituciones de educación superior convocara oficialmente a las universidades e institutos de educación superior a elaborar sus PAI, instrumentos que implantarían la sustentabilidad de forma transversal, pero que no tuvieron la respuesta esperada (Bravo, 2012). Se publicaron pocos PAI y no todos perduraron, aunque surgieron otras iniciativas que no derivaron de un PAI, por lo que se puede advertir que «no existe un modelo común para la organización de la gestión ambiental en las IES» (Sáenz y Benayas, 2015, p. 216), sino que «predomina una gran diversidad de enfoques y prácticas» (Sáenz y Benayas, 2015, p. 218), lo que indica que no se ha alcanzado un acuerdo sobre el establecimiento de políticas universitarias para la sustentabilidad.

Martínez-Fernández y González (2015) apuntan que las iniciativas mexicanas de sistemas de gestión ambiental «distan de ser efectivas en la búsqueda de un cambio articulado de la institución» (p. 70); es decir, se muestran desvinculadas de las tareas sustantivas de educación y difusión de la cultura, lo que sugiere que «las políticas para la sustentabilidad no forman parte del núcleo duro de la agenda institucional y tampoco son de aplicación general a todo el campus» (González et al., 2015, p. 75).

Al respecto, Martínez-Fernández y González (2015) señalan que la escasa movilización de los instrumentos de política universitaria tiene que ver con la complejidad que reporta su elaboración, tanto por su carácter general como por el involucramiento de todas las funciones sustantivas, y el establecimiento de estrategias transversales. Además, sugieren que otro obstáculo para el diseño de un plan transversal es que precisa de la participación de la alta dirección institucional.

En este sentido, no sorprende que una parte importante de los estudios sobre la sustentabilidad en las universidades se dedique a estudiar las políticas universitarias, tanto para dar cuenta de la importancia y dificultades de insertar a la sustentabilidad como eje central en las políticas y prácticas docentes, de investigación, gestión y difusión de la cultura (Alfie-Cohen y Martínez-Fernández, 2015; Martínez-Fernández y González, 2016), como para señalar las dificultades que enfrentan dichas propuestas. Entre estas últimas pueden citarse las convergencias desafortunadas, los objetivos desarticulados y las estrategias laxas (Martínez-Fernández y González, 2015; González et al., 2015).

Con esta revisión se advierte que la sustentabilidad universitaria en México muestra avances en la dimensión «universidad integral». La línea de investigación que documenta la ambientalización del currículo universitario y de la investigación en educación ambiental, con sus avances y contradicciones, refleja una transformación en las funciones sustantivas primordiales de las universidades.

La dimensión de "universidad integrada» también muestra un avance en la línea de investigación que documenta el enverdecimiento de las operaciones del campus. El 
desarrollo de prácticas ambientales concretas es una forma en que la universidad responde a la realidad de su contexto. Estas prácticas expresan el interés por construir nuevas relaciones entre la comunidad universitaria y su medioambiente, y al hacerlo, renuevan la relación con la colectividad social a la que se debe. Sin embargo, esta revisión descubrió que la dimensión «universidad integradora» tiene que fortalecerse; de ello habla la falta de consolidación de la gestión universitaria como un proceso amplio, transversal y ejecutable a través de instrumentos rectores para ofrecer una respuesta sólida y coherente.

La falta de progreso en la dimensión «universidad integradora» podría explicar por qué la sustentabilidad no forma parte de la agenda de las universidades, salvo en honrosas excepciones, o se asume en forma retórica sin atender a las transformaciones que su implantación demanda. Lo anterior repercute en que no se concreten los cambios sustantivos y estructurales, y tampoco se construya coherentemente una relación entre la universidad y su realidad social. El estancamiento de la universidad integradora advierte que la sustentabilidad universitaria enfrenta inercias y resistencias, que González et al. (2015) asemejan al «techo de cristal» de los estudios feministas

\section{CONCLUSIONES}

La sustentabilidad se señala como una vía para remontar la tragedia ambiental contemporánea, y el papel de la universidad es fungir como el faro para guiar a la sociedad en dicho camino. Este trabajo, a partir de una revisión de la literatura en México, mostró sus avances y sus desafíos en tres dimensiones: universidad integral, universidad integrada y universidad integradora.

Los avances más importantes se tienen en la dimensión de la «universidad integral», pues se ha consolidado una línea de investigación que da cuenta de la alineación de las funciones sustantivas universitarias hacia el desarrollo sustentable. No obstante, en esta dimensión es menester atender los desafíos correspondientes a la insuficiente inclusión curricular de la sustentabilidad y la escasez de programas para los educadores ambientales, y superar la negación hacia los aportes de otras disciplinas en el entendimiento de la crisis socioambiental que experimentamos.

La dimensión «universidad integrada» muestra un avance discreto, pues, aunque la universidad es la institución por antonomasia para responder a las necesidades de la sociedad, y la sustentabilidad le plantea una agenda amplia con intervenciones en favor del planeta, las personas y la prosperidad, la respuesta universitaria se ha acotado al despliegue de actividades ambientales para el enverdecimiento de las operaciones en sus campus. La articulación de una respuesta integral, oportuna y creativa sigue siendo una asignatura pendiente para avanzar hacia la sustentabilidad universitaria.

La falta de avances en la dimensión «universidad integradora» repercute en que no se consolide la sustentabilidad como una políti- 
ca de las universidades. Es la dimensión que muestra mayores resistencias a experimentar la transformación profunda que la sustentabilidad demanda. Centrar la atención en aquellos elementos que impiden los cambios y que atentan contra la construcción de una relación coherente entre la universidad y la realidad contemporánea, permite ubicar en los aspectos institucionales y organizacionales tanto obstáculos como facilitadores de la sustentabilidad universitaria.

En términos generales, esta reflexión arroja que el avance en las distintas dimensiones de la sustentabilidad universitaria recae en gran medida en la voluntad política del gobierno universitario, pues son los encargados de propiciar el cambio en sus comunidades y diseñar las estructuras institucionales y organizacionales para solventarlo. El compromiso de los funcionarios universitarios para vislumbrar el futuro de sus instituciones en el camino de la sustentabilidad no es menor y es el único que puede asegurar la convergencia en las dimensiones integral e integrada, al diseñar las directrices y políticas que transformen al campus universitario en un enclave para la sustentabilidad.

\section{REFERENCIAS BIBLIOGRÁFICAS}

Alfie-Cohen, M. y Martínez-Fernández, C. N. (2015). La UAM Cuajimalpa: Reflexiones en su décimo aniversario. Revista de la Educación Superior, 44(176), 37-61. https://doi.org/10.1016/i. resu.2015.12.003

Anzueto, M. (2015). Pride, un modelo probado para contribuir con los objetivos de formación de la educación ambiental en Latinoamérica. Revista Mexicana de Educación Ambiental Jandiekua, 2(4), 39-46. https://bit.ly/3hMirq0

Asociación Nacional de Instituciones de Educación Superior. (2020). Anuario Estadístico de la Población Escolar en Educación Superior. Ciclo Escolar 2019-2020. ANUIES. https://bit.Iy/3izILj8

Ávila, L.E. (2014). Los programas ambientales universitarios en México. Entre el discurso ambiental y los negocios verdes. Sociedad y Ambiente, 1(3), 26-51. https://doi.org/10.31840/sya.v0i3.995

Barnes, P. y Jerman, P. (2002). Developing an environmental management system for a multiple-university consortium. Journal of Cleaner Production, 10(1), 33-39. https://doi.org/10.1016/S09596526(01)00020-8

Bravo, M. T. (2006) Educación superior. En T. Ruge, C. Velasco, J. Reyes, E. Castro y V. Bedoy (Coord.) Estrategia nacional de Educación Ambiental para la Sustentabilidad en México (pp. 89-112). Secretaría de Medio Ambiente y Recursos Naturales; Centro de Educación y Capacitación para el Desarrollo Sustentable. https://bit.ly/2Ux4Uuu 
Bravo, M. T. (Coord.) (2012). Los planes ambientales institucionales en la educación superior en México Construyendo sentidos de sustentabilidad (2002-2007). Asociación Nacional de Universidades e Instituciones de Educación Superior; Secretaría de Medio Ambiente y Recursos Naturales; Universidad Nacional Autónoma de México. https://bit.Iy/3qUluiR

Calixto, R. (2012). Investigación en educación ambiental. Revista Mexicana de Investigación Educativa. 17(55), 1019-1033. https://bit.ly/3hnsCIM

Calixto, R. (2018). El cambio climático en las representaciones sociales de los estudiantes universitarios. Revista Electrónica de Investigación Educativa, 20(1), 122-132. https://doi.org/10.24320/ redie.2018.20.1.1443

Cantú-Martínez, P. C. (2013). Las instituciones de educación superior y la responsabilidad social en el marco de la sustentabilidad. Revista Electrónica Educare, 17(3), 41-55. https://bit.ly/2TH9XbH

Carlson, S. (8 de noviembre de 2015). Whatever happened to the drive for campus sustainability? The Chronicle of Higher Education. https://bit.ly/2UAeJrz

Celaya, A., Luque, D., García, J., Amozurrutia de María, J. A., Preciado, J. M., Laborín, J. y Cabanillas, R. E. (2017). Evaluación de la producción científica de sustentabilidad ambiental en un centro público de investigación (CPI) del Conacyt (1982-2012). Revista de la Educación Superior, 46(182), 89-112. https://doi.org/10.1016/j.resu.2017.04.002

Fuentes, N. y González, H. E. (2016). Ambientalización del currículo universitario: un reto de la ecopedagogía. Tecné, Episteme y Didaxis: TED, (40), 217-234. https://doi.org/10.17227/01203916.6154

González, E. J. y Meira, P. A. (2020). Educación para el cambio climático: ¿educar sobre el clima o para el cambio? Perfiles Educativos, 42(168), 157-174. https://doi.org/10.22201/iisue.24486167e.2020.168.59464

González, E. J., Meira-Cartea, P. y Martínez-Fernández, C. N. (2015). Sustentabilidad y universidad: retos, ritos y posibles rutas. Revista de la Educación Superior, 44(175), 69-93. https://doi.org/10.1016/i. resu.2015.09.002

González, E. J. y Arias, M. A. (2009). La educación ambiental institucionalizada: actos fallidos y horizontes de posibilidad. Perfiles Educativos, 31(124). https://doi.org/10.22201/iisue.24486167e.2009.124.18835

Harring, N., Lundholm, C. y Torbjörnsson, T. (2017). The Effects of Higher Education in Economics, Law and Political Science on Perceptions of Responsibility and Sustainability. En W. Leal, L. Brandli, P. Castro y J. Newman (Eds), Handbook of Theory and Practice of Sustainable Development in Higher Education (Vol. 1, pp. 159-170). Springer. https://bit.ly/3qWFUbU 
Martínez-Fernández, C. y González, E. J. (2015). Las políticas para la sustentabilidad de las Instituciones de Educación Superior en México: entre el debate y la acción. Revista de Educación Superior, 44(174), 61-74. https://doi.org/10.1016/j.resu.2015.06.002

Martínez-Fernández, C. N. y González, E. J. (2016). La sustentabilidad en la Universidad Veracruzana al término del decenio de la educación para el desarrollo sustentable. Revista Iberoamericana de Educación Superior, 7(19), 168-180. https://doi.org/10.22201/iisue.20072872e.2016.19.193

Martínez, M. G. y Juárez, L. G. (2019). Diseño y validación de un instrumento para evaluar la formación en sostenibilidad en estudiantes de educación superior. IE Revista De Investigación Educativa de la REDIECH, 10(19), 37-54. https://doi.org/10.33010/ie rie rediech.v10i19.501

Miranda, L. F., Sánchez, J. O. y Viloria, J. J. (2021). Environmental sustainability in higher education: mapping the field. Revista Electrónica de Investigación Educativa, 23(e09), 1-16. https://doi.org/10.24320/ redie.2021.23.e09.4053

Mora, W. M. (2015). Desarrollo de capacidades y formación en competencias ambientales en el profesorado de ciencias. Tecné, Episteme y Didaxis: Ted, (38), 185-203. https://doi. org/10.17227/01203916.3794

Ortiz, B. (2019). Enfoque sistémico, medio ambiente y educación superior en México. Regiones y Desarrollo Sustentable, 19(37), 129-150. https://bit.ly/3e15BCZ

Ortiz, T. M., Cruz, G. E. y Bello, L. (2019). La representación social de la problemática ambiental en profesores de ingeniería civil, de la Escuela Superior de Ingeniería y Arquitectura del Instituto Politécnico Nacional. Revista de la Educación Superior, 48(190), 85-209. https://doi.org/10.36857/ resu.2019.190.716

Pedraza, Y. (2017). El compromiso ambiental universitario un desafío curricular para trascender. Cadernos cimeac, 7(2), 120-145. https://bit.ly/36lOdVv

Pedraza, Y. (2020). La investigación-acción-participativa para problematizar la ambientalización curricular universitaria. Tecné, Episteme y Didaxis: Ted, (47), 93-109. https://doi.org/10.17227/ted.num47$\underline{9516}$

Porras, Y. A. (2015). Representaciones sociales sobre la crisis ambiental de profesores de química en formación inicial de la Universidad Pedagógica Nacional. Tecné, Episteme y Didaxis: Ted, (38), 3755. https://doi.org/10.17227/01203916.3786

Prado, S. E. y Pérez, E. (2010). Participación estudiantil en programas ambientales en instituciones de educación superior. Perfiles Educativos, 33(134). https://doi.org/gmwf

Rosas, M., Santiago, M. E. y Juárez L. A. (2014). La economía ecológica y solidaria en el currículo del siglo XXI: el caso de la Maestría en Gestión de Proyectos para el Desarrollo Solidario del IPN. Revista de la Educación Superior, 43(170), 89-112. https://doi.org/10.1016/i.resu.2015.02.003 
Sáenz, O. (2015) Diagnósticos nacionales sobre la inclusión de consideraciones ambientales en las universidades de América Latina y El Caribe. Ambiens: Revista Iberoamericana Universitaria en Ambiente, Sociedad y Sustentabilidad, 1(1), 13-36. https://bit.ly/3dZIWIB

Sáenz, O. y Benayas, J. (2015) Ambiente y Sustentabilidad en las Instituciones de Educación Superior en América Latina y El Caribe. Ambiens: Revista Iberoamericana Universitaria en Ambiente, Sociedad y Sustentabilidad, 1(2), 192-220. https://bit.ly/3xwWBx6

Sánchez-Contreras, M. F. y Murga-Menoyo M. A. (2019). El profesorado universitario ante el proceso de ambientalización curricular. Sensibilidad ambiental y práctica docente innovadora. Revista Mexicana de Investigación Educativa, 24(82), 765-787. https://bit.ly/36pzY1K

Saucedo, S. (2015). Diseño y educación ambiental: un primer encuentro. Jandiekua, 2(4), 5-12. https://bit.ly/3qYs2hr

Suasnábar, C., del Valle, D., Didriksson, A. y Korsunsky, L. (2018). Introducción. En C. Suasnábar, D. del Valle, A. Didriksson y L. Korsunsky (Coord.), Balance y desafíos hacia la CRES 2018 (pp. 1728). Instituto de Estudios y Capacitación de la Federación Nacional de Docentes Universitarios (IEC-CONADU); Consejo Latinoamericano de Ciencias Sociales (CLACSO); Universidad Nacional de las Artes (UNA); Universidad Nacional de las Artes. https://bit.ly/3dYBpbL 